\title{
Whole-exome sequencing and neurite outgrowth analysis in autism spectrum disorder
}

\author{
Ryota Hashimoto ${ }^{1,2}$, Takanobu Nakazawa ${ }^{3}$, Yoshinori Tsurusaki ${ }^{4}$, Yuka Yasuda ${ }^{2}$, Kazuki Nagayasu ${ }^{3}$, \\ Kensuke Matsumura ${ }^{5}$, Hitoshi Kawashima ${ }^{6}$, Hidenaga Yamamori ${ }^{2}$, Michiko Fujimoto ${ }^{2}$, Kazutaka Ohi ${ }^{2}$, \\ Satomi Umeda-Yano ${ }^{7}$, Masaki Fukunaga ${ }^{8}$, Haruo Fujino ${ }^{9}$, Atsushi Kasai ${ }^{5}$, Atsuko Hayata-Takano ${ }^{1,5}$, \\ Norihito Shintani ${ }^{5}$, Masatoshi Takeda ${ }^{1,2}$, Naomichi Matsumoto ${ }^{4}$ and Hitoshi Hashimoto ${ }^{1,3,5}$
}

Autism spectrum disorder (ASD) is a complex group of clinically heterogeneous neurodevelopmental disorders with unclear etiology and pathogenesis. Genetic studies have identified numerous candidate genetic variants, including de novo mutated ASD-associated genes; however, the function of these de novo mutated genes remains unclear despite extensive bioinformatics resources. Accordingly, it is not easy to assign priorities to numerous candidate ASD-associated genes for further biological analysis. Here we developed a convenient system for identifying an experimental evidence-based annotation of candidate ASD-associated genes. We performed trio-based whole-exome sequencing in 30 sporadic cases of ASD and identified 37 genes with de novo single-nucleotide variations (SNVs). Among them, 5 of those 37 genes, POGZ, PLEKHA4, PCNX, PRKD2 and $H E R C 1$, have been previously reported as genes with de novo SNVs in ASD; and consultation with in silico databases showed that only HERC1 might be involved in neural function. To examine whether the identified gene products are involved in neural functions, we performed small hairpin RNA-based assays using neuroblastoma cell lines to assess neurite development. Knockdown of 8 out of the 14 examined genes significantly decreased neurite development $(P<0.05$, one-way analysis of variance), which was significantly higher than the number expected from gene ontology databases $(P=0.010$, Fisher's exact test). Our screening system may be valuable for identifying the neural functions of candidate ASD-associated genes for further analysis and a substantial portion of these genes with de novo SNVs might have roles in neuronal systems, although further detailed analysis might eliminate false positive genes from identified candidate ASD genes.

Journal of Human Genetics (2016) 61, 199-206; doi:10.1038/jhg.2015.141; published online 19 November 2015

\section{INTRODUCTION}

Autism spectrum disorder (ASD) is a neurodevelopmental disorder characterized by impairments in social interactions, reduced verbal communication abilities, stereotyped repetitive behaviors and restricted interests. The complex etiology of ASD includes genetic and environmental factors; the high heritability of this disorder supports the presence of a significant genetic contribution. Recent studies have shown that de novo single-nucleotide variations (SNVs) greatly contribute to the risk of several brain disorders, including ASD, schizophrenia and intellectual disability. ${ }^{1-9}$ In particular, numerous de novo SNVs were identified using whole-exome sequencing (WES) of individuals with sporadic ASD. ${ }^{1-3,6-9}$ Among them, several disruptive mutations associated with sporadic ASD were recurrently found in
CHD8, SCN2A, GRIN2B, DYRK1 and SYNGAP1 (for review, see Krumm, N. et al. ${ }^{4}$, Ronemus, M. et al. ${ }^{10}$ ), which have led to a convergence on several functional pathways such as chromatin remodeling, the Wnt signaling pathway and synaptic function; however, the genetic mutations in these genes account for only a small proportion of all cases. To make matters worse, the functions of most of candidate ASD-associated genes, as well as the biological significance of the identified mutations in the central nervous system largely remain elusive despite extensive bioinformatics resources. Accordingly, although the precise functional analysis of each diseaseassociated gene and its mutation is important for understanding the etiology of ASDs, it is not easy to assign priorities to numerous candidate ASD-associated genes for further detailed biological analysis.

\footnotetext{
${ }^{1}$ Molecular Research Center for Children's Mental Development, United Graduate School of Child Development, Osaka University, Osaka, Japan; ${ }^{2}$ Department of Psychiatry, Osaka University Graduate School of Medicine, Osaka, Japan; 3iPS Cell-Based Research Project on Brain Neuropharmacology and Toxicology, Graduate School of Pharmaceutical Sciences, Osaka University, Osaka, Japan; ${ }^{4}$ Department of Human Genetics, Yokohama City University Graduate School of Medicine, Yokohama, Japan; ${ }^{5}$ Laboratory of Molecular Neuropharmacology, Graduate School of Pharmaceutical Sciences, Osaka University, Osaka, Japan; ${ }^{6}$ Genomic Science Laboratories, Sumitomo Dainippon Pharma Co., Ltd., Osaka, Japan; ${ }^{7}$ Department of Molecular Neuropsychiatry, Osaka University Graduate School of Medicine, Osaka, Japan; ${ }^{8}$ Division of Cerebral Integration, National Institute of Physiological Sciences, Aichi, Japan and ${ }^{9}$ Graduate School of Human Sciences, Osaka University, Osaka, Japan

Correspondence: Dr R Hashimoto, Molecular Research Center for Children's Mental Development, United Graduate School of Child Development, Osaka University, D3, 2-2, Yamadaoka, Suita, Osaka 565-0871, Japan.

E-mail: hashimor@psy.med.osaka-u.ac.jp

or Dr T Nakazawa, iPS Cell-Based Research Project on Brain Neuropharmacology and Toxicology, Graduate School of Pharmaceutical Sciences, Osaka University, 1-6 Yamadaoka, Suita, Osaka 565-0871, Japan.

E-mail: takanobunakazawa-tky@umin.ac.jp

Received 2 October 2015; revised 23 October 2015; accepted 28 October 2015; published online 19 November 2015
} 
To circumvent these problems, developing a rapid and convenient system for identifying an experimental evidence-based annotation of the numerous candidate ASD-associated genes is imperative.

In this study, we performed trio-based WES in ASD patients and identified 37 genes with de novo SNVs. Then, we developed a neuroblastoma cell-based rapid and convenient screening system for elucidating the functions of these gene products in the central nervous system. Of the 14 genes examined with this screening system, 8 were identified as regulators of neurite outgrowth, which is a significantly higher proportion than expected from simply using the gene ontology databases, although further detailed analysis might eliminate false positive genes from identified candidate ASD genes.

\section{MATERIALS AND METHODS}

Following a description of the study, written informed consent was obtained from each subject. This study was carried out in accordance with the World Medical Association's Declaration of Helsinki and was approved by the Research Ethics Committee in Osaka University. All recombinant DNA experiments were reviewed and approved by the Gene Modification Experiments Safety Committee in Osaka University.

\section{Subjects}

The cohort consisted of 30 patients with ASD (21 males and 9 females; mean age \pm s.d., $22.1 \pm 7.8$ years) and their parents (mean age \pm s.d. at sampling, father: $54.5 \pm 6.9$ years, mother: $52.3 \pm 7.7$ years; mean age \pm s.d. at conception of proband, father: $31.9 \pm 3.9$ years, mother: $28.9 \pm 3.5$ years; for details, see Supplementary Materials and Methods). All participants are Japanese and were recruited from either outpatient or inpatient services at the Osaka University Hospital. We obtained participant data from the research bio-resource of the Human Brain Phenotype Consortium in Japan (http://www.sp-web.sakura.ne. jp/consortium.html). Each ASD patient was diagnosed by at least two trained child psychiatrists according to the Diagnostic and Statistical Manual of Mental Disorders, $5^{\text {th }}$ edition (DSM-5). The patients were assessed through unstructured or semi-structured behavioral observation and interviews with the patients and their parents or caregivers as previously described. ${ }^{11-13}$ In addition, the Autism Diagnostic Interview-Revised, ${ }^{14}$ the Pervasive Developmental Disorders Autism Society Japan Rating Scale ${ }^{15}$ and the Japanese version of Asperger's Questionnaire ${ }^{16}$ were used to assist in the evaluation of ASD-specific behaviors and symptoms. Intelligence quotient (IQ) data for the subjects were collected using the Japanese version of the full-scale Wechsler Adult intelligence Scale-III $(n=23),{ }^{17}$ the full-scale Wechsler Intelligence Scale for ChildrenThird Edition $(n=5),{ }^{18}$ or the Japanese version of the NART 50, which can measure estimated IQ scores $(n=2) .{ }^{19}$ Among the patients with ASD, 2 patients had low IQ scores $(\leqslant 69), 2$ patients had borderline IQ scores (70-79), 22 patients had normal IQ scores (80-119) and 4 patients had superior IQ scores $(\geqslant 120)$ (for detail, Supplementary Materials and Methods). The mean full-scale IQ \pm s.d. of all patients with ASD was $100.9 \pm 19.8$.

\section{DNA preparation and WES}

Peripheral blood samples were collected from the subjects. Genomic DNA was extracted from whole blood using a QIAamp DNA Blood Maxi Kit (Qiagen, Hilden, Germany). Parentage was confirmed using nine polymorphic microsatellite markers (D1S450, D1S206, D5S630, D5S410, D9S285, D9S1776, D13S217, D14S276 and D14S985). The amplicons were separated on a Genetic Analyzer 3500 (Life Technologies Inc., Carlsbad, CA, USA), and analyzed using GeneMapper software version 4.1 (Life Technologies Inc.). Trio-based WES was performed in every family. Genomic DNA was captured with the SureSelect Human All Exon v.4 Kit (Agilent Technologies, Santa Clara, CA, USA) and sequenced with four samples per lane on an Illumina HiSeq 2000 (Illumina, San Diego, CA, USA) with 101-bp paired-end reads. Image analysis and base calling were performed by Sequencing Control Software with Real-Time Analysis and CASAVA software v.1.8 (Illumina). Exome data processing, variant calling and variant annotation were performed as previously described. ${ }^{20-23}$ Mapping to human genome hg19 was performed using Novoalign (Novocraft Technologies, Selangor, Malaysia). Aligned reads were processed by Picard to remove PCR duplicates. Variants were called using the Genome Analysis Toolkits v.1.6-5 (GATK). ${ }^{20}$ Called SNVs, insertions and deletions (indels) were annotated using ANNOVAR. ${ }^{23}$ From the total variants within exons and $\pm 10 \mathrm{bp}$ of intronic regions from exon-intron boundaries, those registered in dbSNP135, ESP 5400 (http://evs.gs.washington.edu/EVS/), 1000 Genomes (http://www.1000genomes.org/), the Human Genetic Variation Database as a reference database of genetic variations in 1208 Japanese controls (http://www.genome.med.kyoto-u.ac.jp/SnpDB) ${ }^{24}$ or our in-house (exome data from 408 individuals) databases at the Yokohama City University School of Medicine and those located with segmental duplications were removed. SNVs detected by WES were confirmed by Sanger sequencing. Primer sequences are available in Supplementary Table S1. The details of the PCR conditions are available on request. Our human genome researches and gene analysis researches were all performed under the Ethical Guidelines for Human Genome/Gene analysis Research, Ministries of Education, Culture, Sports, Science, and Technology, Health, Labor and Welfare, and Economy, Trade and Industry. According to the Guidelines, the research institution and its joint research institution approved by the ethics review committee but not thirdparty institutions can only deal with any potential personally identifiable information. In conformity to the Guidelines, we did not obtain the subjects' consent for the disclosure of any potential personally identifiable information. Accordingly, we could not disclose any potential personally identifiable information such as whole-exome sequence data of each subject to thirdparty institutions or public databases.

\section{Cells}

Mouse neuroblastoma Neuro2a cells were cultured in Advanced Dulbecco's Modified Eagle Medium (DMEM)/F12 (Life Technologies) supplemented with $10 \%$ fetal bovine serum, GlutaMAX (Life Technologies), and non-essential amino acid solution (Life Technologies). Neuro2a cells were infected with recombinant lentivirus expressing small hairpin (shRNA) at a multiplicity of infection of $\sim 40$. Two days after infection, the cells were treated with $30 \mu \mathrm{M}$ retinoic acid in Advanced DMEM/F12 supplemented with $0.2 \%$ fetal bovine serum to induce neuronal differentiation associated with neurite outgrowth. After $24 \mathrm{~h}$, cell morphology was observed (Supplementary Figure S1). Lenti-X $293 \mathrm{~T}$ cells (Clontech, Mountain View, CA, USA) were maintained in DMEM containing $10 \%$ fetal bovine serum for lentivirus production. ${ }^{25,26}$

\section{Preparation of recombinant lentivirus}

Recombinant lentivirus was prepared essentially as described previously ${ }^{27}$ with some modifications. Briefly, $5.2 \mu \mathrm{g}$ of pNHP, $2.1 \mu \mathrm{g}$ of pHEF-VSVG, $0.43 \mu \mathrm{g}$ of pCEP4-tat and $2.6 \mu \mathrm{g}$ of MISSION shRNA construct (Supplementary Table S2, Sigma-Aldrich, St Louis, MO, USA) were transfected into Lenti-X 293T cells (Clontech) in one 10-cm dish using polyethylenimine (Polyethylenimine 'Max', Mw 40 000; Polysciences, Warrington, PA, USA). After 16-18 h of incubation, the culture supernatant was replaced with fresh media. After $24 \mathrm{~h}$ of incubation, the culture supernatant was collected, filtered through a syringe filter with a polyvinylidene fluoride membrane $(0.45-\mu \mathrm{m}$ pore size, Millex-HV, Merck Millipore, Billerica, MA, USA) and stored in aliquots at $-80^{\circ} \mathrm{C}$. Titration of recombinant lentiviruses was performed with Lenti-X qRT-PCR titration kit (Clontech). An shRNA sequence that does not target any mouse genes (SHC002, Sigma-Aldrich) was used as a control.

\section{Quantification of neurite length in Neuro2a cells}

Before the experiment, we confirmed that almost all cells were positive for green fluorescent protein expression with lentivirus expressing green fluorescent protein at multiplicity of infection of $\sim 40$ (data not shown). Thus, lentiviruses expressing shRNA against candidate ASD-associated genes were used at multiplicity of infection of $\sim 40$. Neuro2a cells were fixed with $4 \%$ paraformaldehyde for $10 \mathrm{~min}$ at room temperature, permeabilized with Triton X-100 and incubated with Hoechst 33258 dye (Calbiochem, San Diego, CA, USA) to identify nuclei and Alexa Fluor 546-phalloidin (Molecular Probes, Eugene, OR, USA) to identify cell bodies and neurites. For automated image analysis, images of Neuro2a cells were acquired using a ToxInsight automated microscope (Thermo Scientific, Waltham, MA, USA). Neurite number per cell, total neurite length per cell, neurite length per neurite and the longest neurite 
Table 1 Mutations with possible contributions to de novo ASD risk

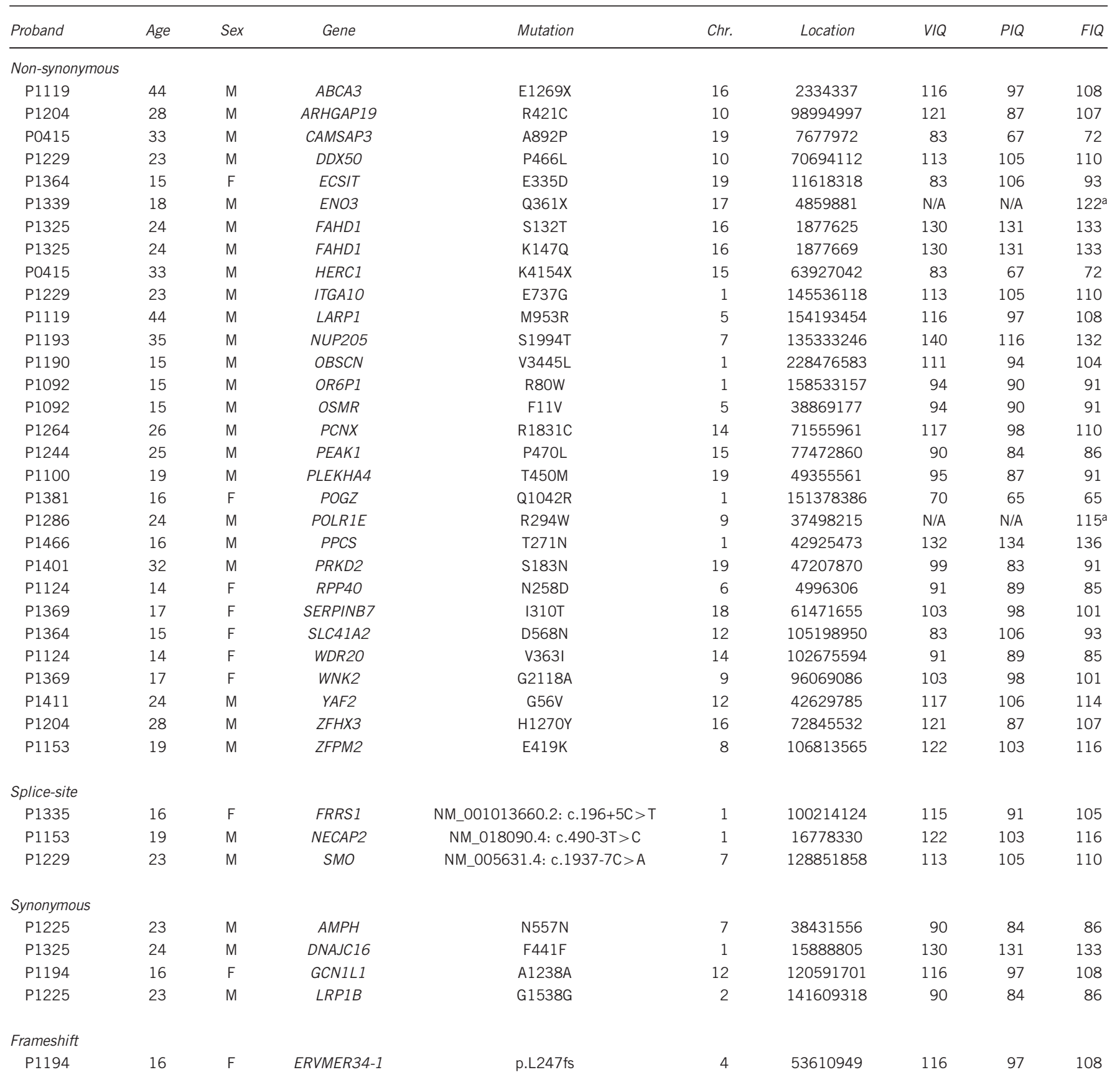

Abbreviations: ASD, autism spectrum disorder; Chr., chromosome; FIQ, full scale IQ; N/A, not applicable; PIQ, performance IQ; VIQ, verbal IQ.

a Japanese adult reading test.

length were automatically measured. Approximately 9000-15000 cells were observed for each construct (see Figure legends). The data presented are from three independent experiments performed in triplicate.

\section{Rescue experiments for the neurite growth assay}

Human PRKD2 and PPCS complementary DNAs, purchased from Origene (Rockville, MD, USA), were amplified via PCR and subcloned into each MISSION shRNA construct. Recombinant lentiviruses expressing shRNAresistant human PRKD2 and shRNA against PRKD2 or expressing shRNAresistant human PPCS and shRNA against PPCS were prepared as described above. Quantification of neurite length in Neuro2a cells expressing these recombinant lentiviruses were performed as described above.

\section{Reverse transcription-PCR analysis}

Total RNA was isolated from Neuro2a cells and reverse transcribed with Superscript III (Life Technologies). ${ }^{28}$ Real-time PCR was performed with SYBR premix EX Taq (Takara Bio Inc., Shiga, Japan) on a CFX96 real-time PCR detection system (Bio-Rad Laboratories, Hercules, CA, USA) according to the supplier's protocol. Intron-spanning primer sets were used (primer sequences are available on request). The expression levels for each gene were normalized to that of GAPDH and were determined according to the 2- $\Delta \Delta \mathrm{Ct}$ method.

\section{Gene annotation}

Gene annotations were interrogated using AmiGO2 (http://amigo2. berkeleybop.org/amigo). The GO annotation terms related to the central 
Table 2 Possible function of identified genes in the central nervous system

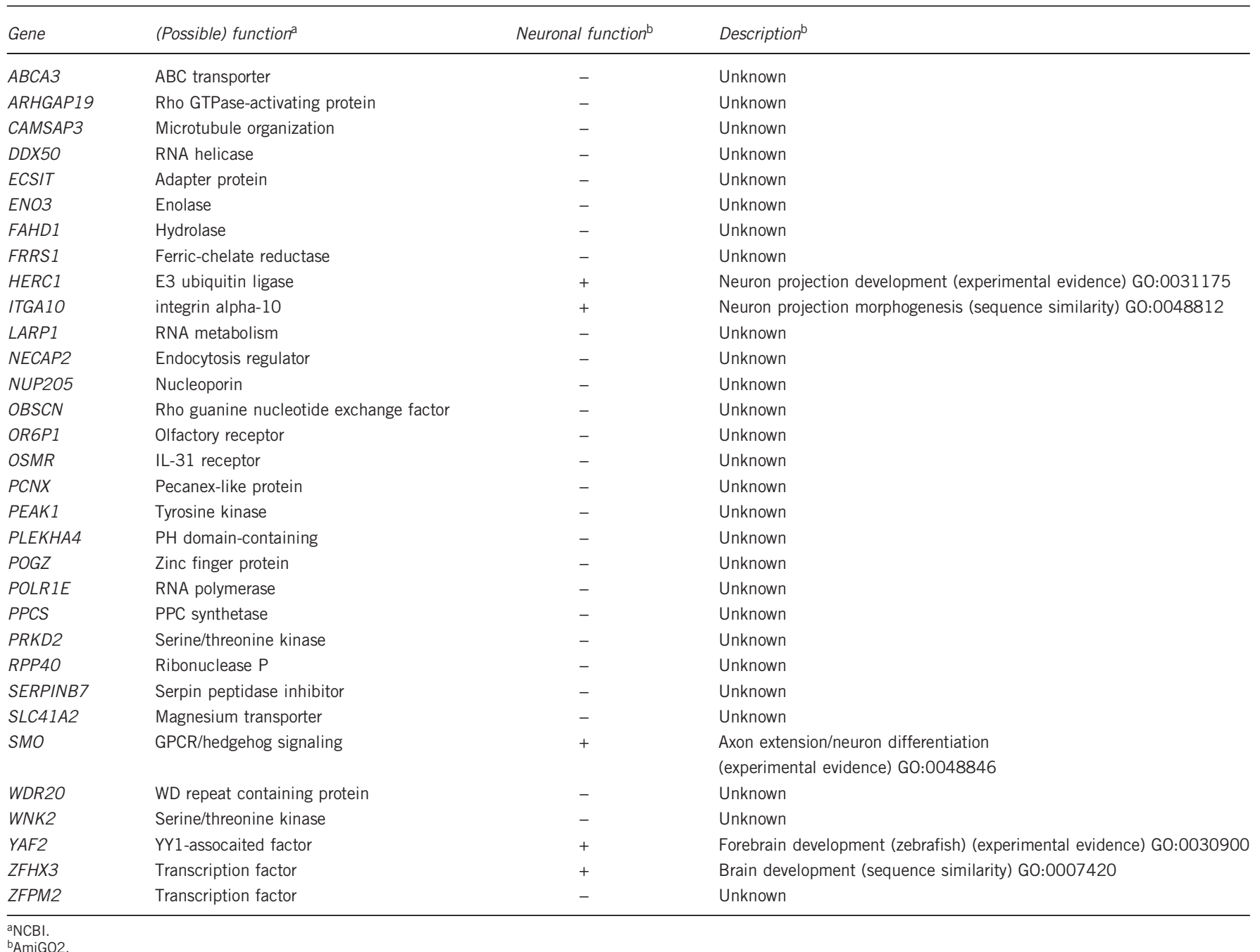

nervous system function, including neurite outgrowth were manually searched for each gene.

\section{Statistical analyses}

Statistical analysis was performed using one-way analysis of variance, or Fisher's exact test, as indicated. All $P$-values reported are two-tailed. The level of significance was set at $P<0.05$.

\section{RESULTS}

\section{WES of 30 sporadic autism trios}

We selected 30 sporadic autism trios from human brain phenotype consortium in Japan (Supplementary Materials and Methods). We performed trio-based WES and validated 38 de novo events, 37 SNVs and one small deletion (Supplementary Materials and Methods). These SNVs included 3 nonsense, 1 frameshift, 27 non-synonymous, 4 synonymous and 3 possible splice-site mutations (Table 1). The observed point-mutation rate in coding sequences was $\sim 1.2$ events per trio, which is similar to that observed in previous studies indicating increased sensitivity. ${ }^{7,8}$ Because the possible functional impact of one frameshift SNV in a retrovirus gene and four synonymous SNVs is low, we selected 33 SNVs (3 nonsense, 27 non-synonymous and 3 possible splice-sites) in 32 genes for further analysis (Table 1). The 33 variants were confirmed by Sanger sequencing (Supplementary
Table S1 and data not shown). The possible function, as well as neuronal function of these 32 gene products is shown in Table 2. Five of those 32 genes, POGZ, PLEKHA4, PCNX, PRKD2 and HERC1, have been previously reported as genes with de novo SNVs in ASD. ${ }^{1-3,6-9}$

The strategy for gene knockdown-based assignment of priorities to the identified disease-related genes for further detailed analysis Recent genetic analyses have generally identified many disease-related genes, most of which have functions that are largely unknown, making further molecular etiological analysis difficult. Thus, it is important to develop a screening system with which we can rapidly and conveniently assign priorities to the number of candidate genes for further detailed analysis. One possible criterion is that autism-associated gene should be expressed in the brain; however, almost identified 32 genes except for SERPINB7 are expressed in the brain (GTEx Project (http://www.gtexportal.org/home/)). Another possible criterion is that autism-associated gene products should have neuronal functions. To examine whether the identified genes with de novo SNV in autism are involved in neural functions, we focused on neurite development in neuronal cells. Neurite development is commonly used for monitoring early neuronal differentiation and is one of the most important events in the formation of neural circuits. ${ }^{29}$ In this study, we used mouse neural crest-derived Neuro2a neuroblastoma cells, which have been 
a

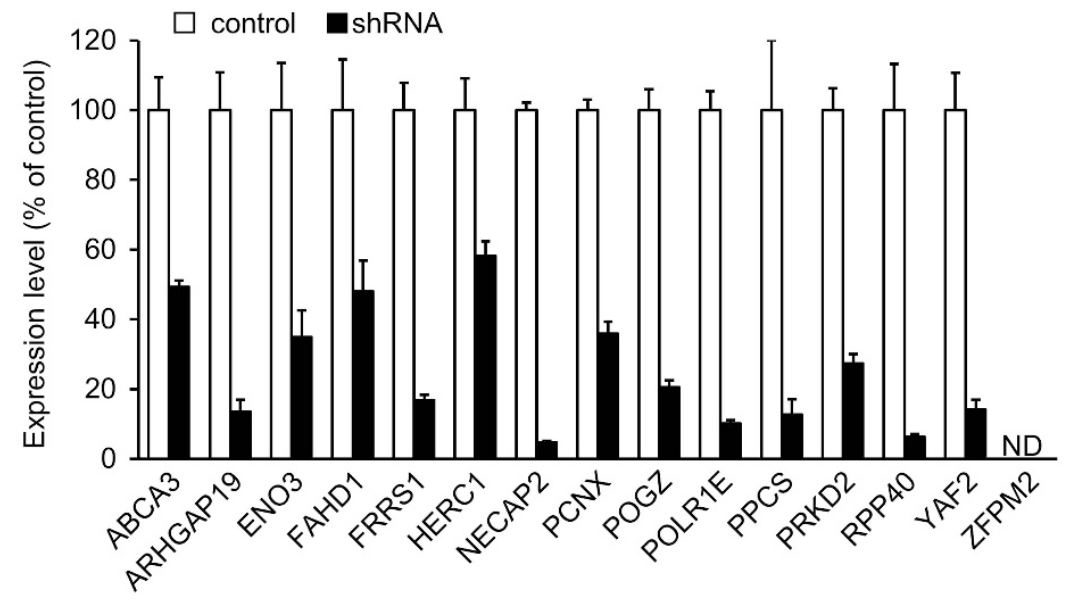

b

\begin{tabular}{cccccc}
$\begin{array}{c}\text { Neurite number } \\
\text { /cell }\end{array}$ & \multicolumn{2}{c}{$\begin{array}{c}\text { Neurite length } \\
\text { /cell }(\mu \mathrm{m})\end{array}$} \\
0.0 & 1.0 & 2.0 & 0.0 & $20.0 \quad 40.0$
\end{tabular}

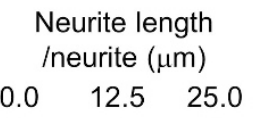
Longest neurite length $(\mu \mathrm{m})$
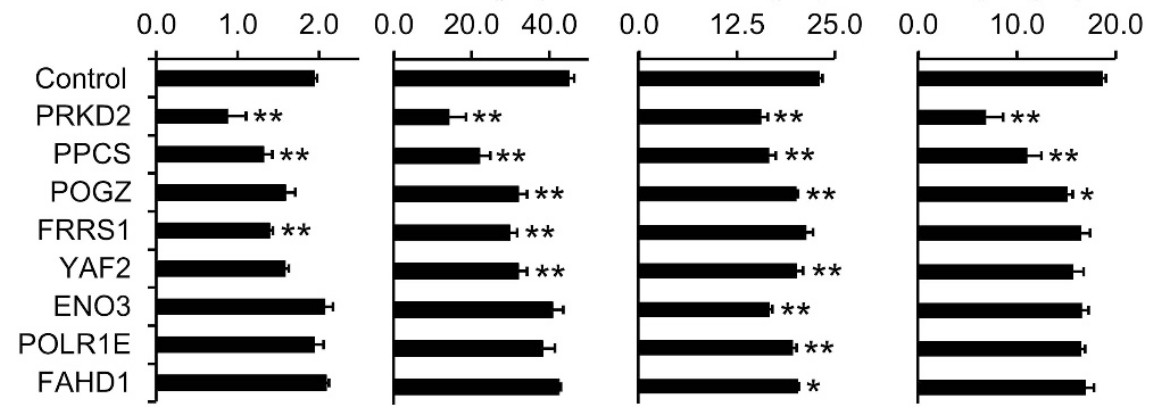

Figure 1 The lentivirus-mediated shRNA knockdown of candidate susceptible genes impaired neurite outgrowth in Neuro2a cells. (a) Decreased expression of the candidate susceptible genes by shRNA knockdown in Neuro2a cells. Neuro2a cells were infected with recombinant lentivirus expressing shRNA against the indicated genes. Two days after infection, total RNA was isolated from Neuro2a cells and reverse transcribed. Real-time PCR was performed with the SYBR system with self-designed primer sets. The expression levels for each gene were normalized to that of GAPDH, which were determined according to the 2- $\Delta \Delta$ Ct method. Note that the shRNA sequence that does not target any mouse genes (Sigma-Aldrich) was used as a control. (b) The lentivirus-mediated shRNA of the indicated genes impaired retinoic acid-induced neurite outgrowth in Neuro2a cells. Hoechst 33258 dye and Alexa Fluor 546-phalloidin were used to identify nuclei and neurites, respectively. Each parameter was automatically measured using ToxInsight (Thermo Scientific). All data are expressed as the mean \pm s.e.m. ${ }^{*} P<0.05,{ }^{*} P<0.01$, one-way ANOVA (vs control). mock, $n=12951$ (average value); PRKD2, $n=11933 ;$ PPCS, $n=9442 ;$ POGZ, $n=11$ 368; FRRS1, $n=9890$; YAF2, $n=9892$; ENO3, $n=15309$; POLR1E, $n=10762 ;$ FAHD1, $n=10122$. Note that statistical analysis was performed using the average value from each experiment $(n=3)$. ANOVA, analysis of variance; shRNA, small hairpin.

extensively used to study neuronal differentiation and its associated signaling pathways. A useful characteristic of Neuro2a cells is their ability to develop relatively simple neurites within a few days, which serves a well-established model system for the study of neurite development of central nervous system neurons. ${ }^{29}$ Using the simple system, we easily determine that the candidate ASD-associated gene products can possibly be involved in neurite development, one of the important aspects of the central nervous system development and function. The system focuses only in neurite development, which gives us fragmentary information of the possible neural function of each gene product, however, the information is enough to drive us to further analyze the function of the gene product in detail. The strategy for gene knockdown-based assignment of priorities of the identified autism-related genes is summarized in Supplementary Figure S2. We searched a commercial shRNA library (MISSION TRC shRNA library SP1, Sigma-Aldrich) for the 32 target genes and found that the library offered 20 validated shRNAs for 15 of those genes (Supplementary Table S2). To maintain the rapid and convenient screening, the remaining 17 genes, for which validated shRNA constructs were not available from the library, were not subjected to further study.
Identification of neural functions of the autism-related genes in mouse neuroblastoma Neuro2a cells

We first tested the knockdown efficiency of the 20 commercially available validated shRNA constructs for the 15 genes and found that the validated shRNA constructs effectively decreased their target RNAs, except for that targeting ZFPM2, which was not detected in Neuro2a cells (Figure 1a and Supplementary Table S2). For FRRS1, POGZ, POLRE1, PPCS and PRKD2, we examined two validated shRNA constructs for knockdown efficiency and chose the more effective one for further morphological studies (data not shown). We then performed shRNA-based assays using Neuro2a cells, which are well known to undergo neural differentiation and extend bidirectional and multidirectional process outgrowth on retinoic acid treatment. ${ }^{29}$ The measured common parameters included the following: neurite number per cell; neurite length per cell; neurite length per neurite; and the length of the longest neurite. Before our experiments, we confirmed that retinoic acid treatment increased the number of neurites and the total length of neurites in Neuro2a cells (Supplementary Figure S1). We compared cells infected with lentiviruses expressing validated shRNA vs cells infected with lentiviruses expressing control shRNA under retinoic acid-differentiated 
conditions for a variety of morphological changes. The shRNAmediated knockdown of these genes did not affect cell viability (data not shown). We analyzed 9000-15000 cells for each construct and found that knockdown of 8 out of 14 genes significantly decreased at least 1 parameter: retinoic acid-induced neurite outgrowth was decreased (Figure $1 \mathrm{~b}$ and Supplementary Table S3). Among these genes, knockdown of PRKD2 or PPCS markedly impaired neurite outgrowth (Figure 2). In addition, knockdown of the other six genes did not significantly affect neurite outgrowth (Supplementary Table S3). These results suggest that the identified eight gene products, PRKD2, PPCS, POGZ, FRRS1, YAF2, ENO3, POLR1E and FAHD1, may be candidate regulators of neurite development in neurons in the central nervous system. We then performed a rescue experiment of PRKD2 and PPCS, disruption of which markedly impaired neurite outgrowth (Figure 2). We found that overexpression of PRKD2 but not PPCS rescued the impaired neurite outgrowth (Figure 3 and data not shown). These results suggest either that the impaired neurite outgrowth by shRNA-mediated knockdown of PPCS may be a false positive results or that overexpressed PPCS cannot substitute the endogenous function of PPCS.

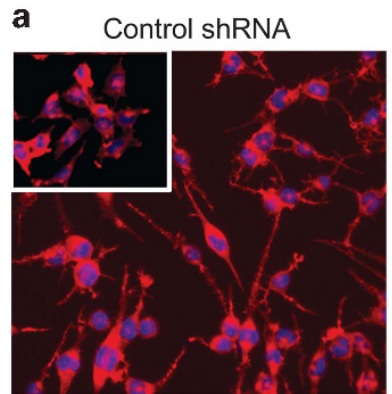

PRKD2 knockdown

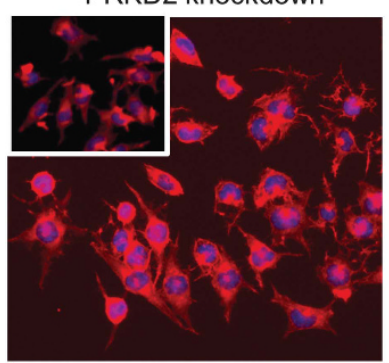

PPCS knockdown

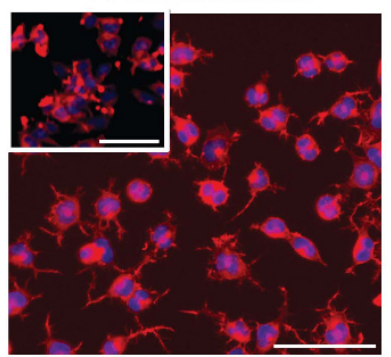

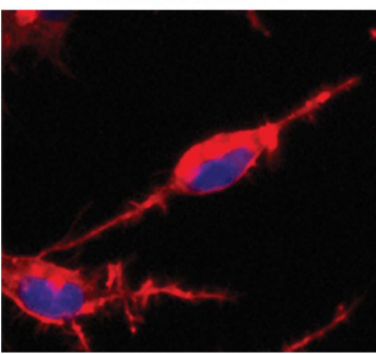

PRKD2 knockdown

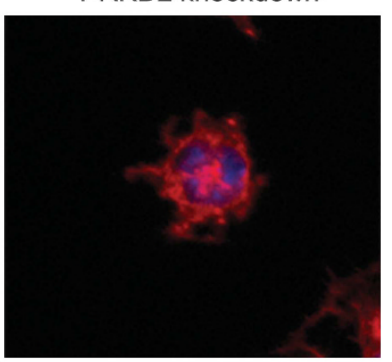

PPCS knockdown

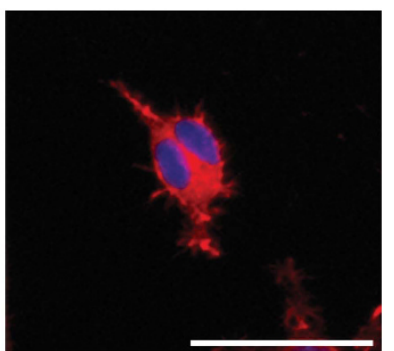

b Control shRNA

Figure 2 The lentivirus-mediated shRNA of PRKD2 or PPCS severely impaired neurite outgrowth in Neuro2a cells. (a) Representative images of retinoic acid-treated Neuro2a cells. Hoechst 33258 dye and Alexa Fluor 546-phalloidin were used to identify nuclei and neurites, respectively. Insets, knocked down cells without retinoic acid treatment. Scale bars, $100 \mu \mathrm{m}$. (b) Representative higher magnification images of retinoic acidtreated Neuro2a cells. Hoechst 33258 dye and Alexa Fluor 546-phalloidin were used to identify nuclei and neurites, respectively. Scale bars, $50 \mu \mathrm{m}$. shRNA, small hairpin.

\section{DISCUSSION}

For the comprehensive analysis of a large number of candidate genes identified by genetic studies, it is necessary to make biological sense of these genes in the central nervous system and to identify convergent signaling pathways in which multiple candidate genes are involved; however, molecular analysis of the function of each individual gene product in the central nervous system generally requires substantial effort. To circumvent this problem, we developed a high-throughput post-genomics screening method with which we can easily predict the neuronal function of the candidate gene products and assign priorities to the number of candidate genes for detailed analysis. We interrogated the annotations of the identified 32 disease susceptibility genes with a Gene Ontology browser (AmiGO2) and found that five of those genes, HERC1, ITGA10, SMO, YAF2 and ZFHX3, are involved in neuronal functions such as neurite outgrowth (Table 2). Our current screening system identified 7 (in the case of excluding PPCS) to 8 (including PPCS) out of the 14 genes examined $(50 \sim 57.1 \%)$ as regulators of neurite development, which is 3.2 3.7-fold higher than that expected from all of AmiGO2 (5 out of 32 genes, $15.6 \%$; $P=0.010$, Fisher's exact test). The system focuses only in neurite development in Neuro2a cells, which gives us fragmentary information of the possible neural function of each gene product, however, the information is enough to drive us to further analyze the function of the gene product in detail. Thus, the Neuro2a cell-based rapid and convenient screening of ASD genes can be valuable for identifying the ASD-related neural functions of enormous pools of candidate genes identified by genetic analyses. Further detailed analysis might eliminate false positive genes from identified candidate ASD genes in our current screening.

In this study, we performed WES of 30 individuals with sporadic ASD and their parents, identified 37 genes with 38 de novo SNVs, and selected 33 SNVs (3 nonsense, 27 non-synonymous and 3 possible splice site) in 32 genes for analysis. Five of those 32 genes, POGZ, PLEKHA4, PCNX, PRKD2 and HERC1, have been previously reported as genes with de novo SNVs in ASD. ${ }^{1-3,6-9}$ A previous study has shown that HERC1 regulates Purkinje cell physiology in the cerebellum; ${ }^{30}$ however, the functions of most of these gene products in the brain had remained unclear despite extensive bioinformatics resources. Using our system, we found that POGZ and PRKD2 regulate neural functions, such as neurite development. Further comprehensive analysis of these recurrently mutated gene products in neural development will unravel the molecular etiology of ASD. In addition to the recurrently mutated genes identified in our study, there are several recurrent disruptive mutated genes, such as CHD8, SCN2A, and DYRK1. ${ }^{4,10}$ It will also be interesting to examine the functions of these gene products in neurite development using our system.

Proper development of axons and dendrites is critical for the anatomical connectivity of brain regions and leads to functional neural circuit formation; precise axon and dendrite development is a biological basis for higher brain function. The elaboration of axons and dendrites is a highly dynamic process that is regulated by the combination of many genes. ${ }^{31}$ ASDs are often associated with altered synaptic connectivity and plasticity, resulting in marked disruptions in information processing and cognition. ${ }^{2}$ Neuropathologically, in ASD patients, the development of axons and dendrites is impaired in multiple brain areas associated with cognitive and behavioral functioning, including the prefrontal cortex..$^{32-34}$ Several ASD-associated gene products such as EPAC2, TAOK2 and PTEN are involved in the maintenance of axonal and dendritic growth. ${ }^{34-38}$ We found that shRNA-mediated knockdown of PRKD2 or PPCS drastically impaired neurite growth in Neuro2a cells, suggesting that these gene products 


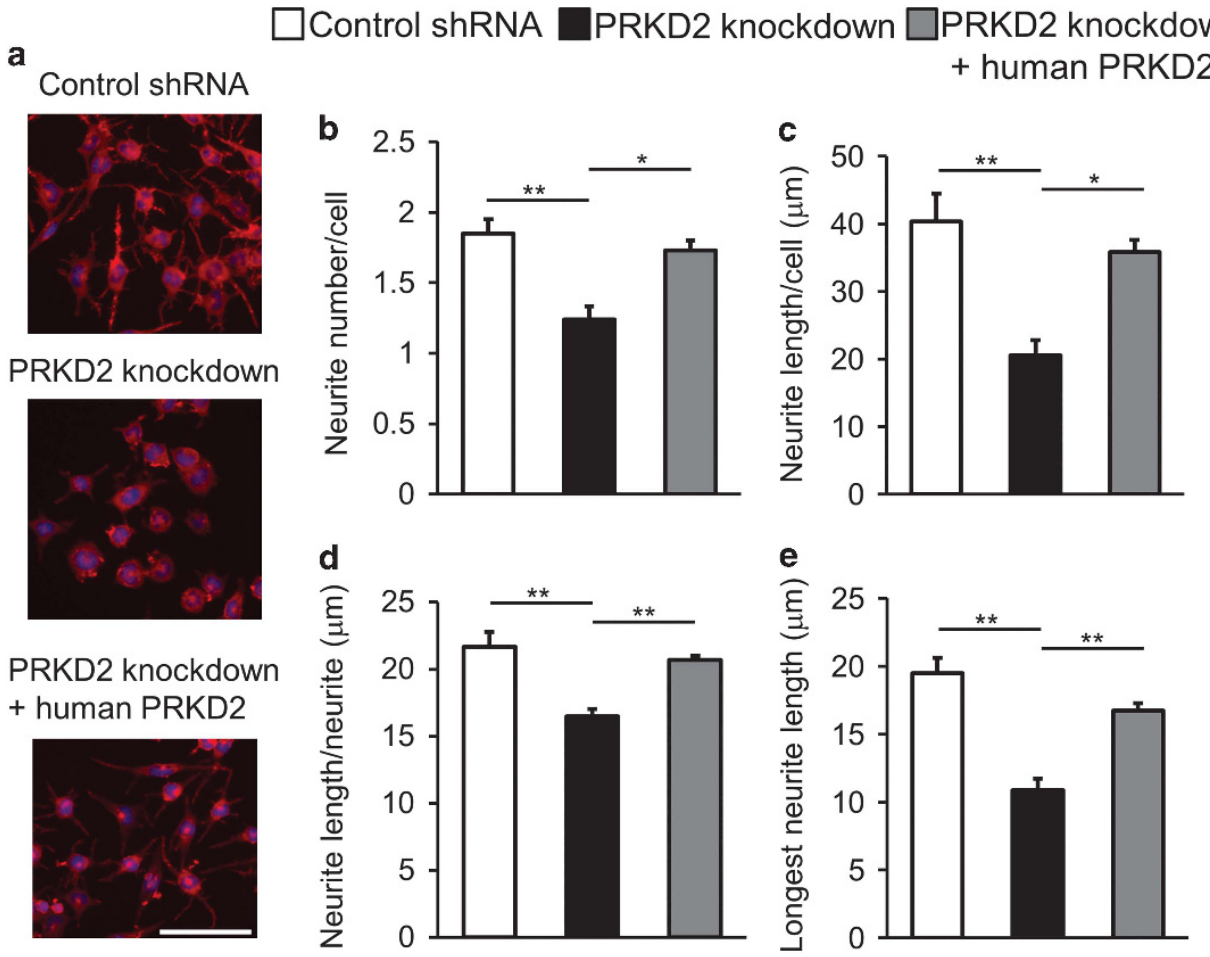

Figure 3 Overexpression of human PRKD2 rescued the PRKD2 knockdown-mediated impaired neurite outgrowth in Neuro2a cells. (a) Representative images of retinoic acid-treated Neuro2a cells. Hoechst 33258 dye and Alexa Fluor 546-phalloidin were used to identify nuclei and neurites, respectively. Scale bars, $100 \mu \mathrm{m}$. (b-e) Quantification of retinoic acid-induced neurite outgrowth in Neuro2a cells. Each parameter was automatically measured using Toxlnsight (Thermo Scientific). All data are expressed as the mean \pm s.e.m. ${ }^{*} P<0.05,{ }^{*} P<0.01$, one-way ANOVA (vs control). Control shRNA, $n=11179$ (average value); PRKD2 knockdown, $n=10$ 069; PRKD2 knockdown+human PRKD2, $n=12532$. Note that statistical analysis was performed using the average value from each experiment $(n=3)$. ANOVA, analysis of variance.

have important roles in dendrite development in neurons. Although the effects were not as marked, the shRNA-mediated knockdown of the remaining six genes also impaired neurite development. A more comprehensive analysis of the molecular mechanisms of axon and dendrite development is important for understanding the etiology of ASDs.

The current study has several limitations as follows:

1. The screening method lacks the positive controls (for example, robust ASD-associated genes such as CHD8 and MEPC2) and negative controls (for example, genes with de novo loss-of-function mutations in healthy siblings in previously published studies). Because of the lack of these important factors, we cannot definitively claim whether the screening system is valid or not.

2. The identified mutations may cause a variety of effects on protein functions, such as loss of function, gain of function, dominant negative suppression of the wild-type protein, or acquisition of a new function. Therefore, the identified mutations cannot be mimicked by simple knockdown experiments.

3. It is important to perform the knockdown experiments with $\sim 50 \%$ knockdown efficiency for examining the putative loss-of-function mutations. Furthermore, the screening method lacks the rescue experiments.

4. In the screening method, we selected the candidate ASD genes for the neurite outgrowth assay in a nonrandom manner.

Thus, the data on sensitivity and specificity seems lacking; further detailed analysis might eliminate false positive genes from the candidate ASD genes, resulting in identifying causative ASD genes.
Given that recent studies argue that true ASD-associated exome SNVs make up $\sim 3-5 \%$ of ASD (for review, see Krumm, N. et al. ${ }^{4}$, Malhotra, D. et al. ${ }^{5}$, Ronemus, M. et al. ${ }^{10}$ ), the number of causative ASD genes among the 30 trios will be only one to two. Although the number of causative ASD genes is expected to be low, the other identified de novo SNVs may also be associated with risk for ASD.

Our current neurobiological screening system using neuroblastoma cell lines focuses only in neurite development, which gives us fragmentary information of the possible neural function of each gene product. It is also important to examine the precise role of the identified candidate gene products in various neural functions, including synapse development and function as well as neurite development. Furthermore, although our system can biologically select genes involving neural function from a vast number of candidate ASD-associated genes for further detailed analysis, the limitation of this study is that we are unable to identify the biological significance of the SNVs using our current screening system. It is important to develop a new system in which we can rapidly examine the biological significance of the SNVs identified by WES of patients with ASD in the near future.

\section{CONFLICT OF INTEREST}

The authors declare no conflict of interest.

\section{ACKNOWLEDGEMENTS}

We thank the patients and their families for participating in this study. This work was supported by research grants from the Japanese Ministry of Health, Labor and Welfare (H26-seishin-ippan-012) (RH), (H26-itaku-(nan)-ippan-083) (NM); Grants-in-Aid for Scientific Research (B) (22390225) (RH), (A) 
(24249019) (NM), (C) (25460100) (AH-T) and (B) (262930200) (HH); and Grants-in-Aid for Challenging Exploratory Research (23659565) (RH) and (26670122) (HH) from Japan Society for the Promotion of Science; Grant-inAid for the Funding Program for Next Generation World-Leading Researchers (LS081) (HH); Grant-in-Aid for the Program for Advancing Strategic International Networks to Accelerate the Circulation of Talented Researchers (S2603) (HH); Grant-in-Aid for Scientific Research on Innovative Areas (Comprehensive Brain Science Network) (RH); Grants-in-Aid for Scientific Research on Innovative Areas (25129704) (YY) and (24118007) (NM) from The Ministry of Education, Culture, Sports, Science, and Technology, the fund for Creation of Innovation Centers for Advanced Interdisciplinary Research Areas Program in the Project for Developing Innovation Systems from the Japan Science and Technology Agency (NM); and grants for research from the Uehara Memorial Foundation, Japan ( $\mathrm{HH}$ and $\mathrm{RH}$ ), the Brain Science Program of National Institutes of Natural Sciences (BS261003) (RH), the Program for Creating Future Wisdom, Osaka University selected in 2014 (TN), Takeda Science Foundation (TN, RH and NM), and the Japan Foundation for Neuroscience and Mental Health (RH).

1 Iossifov, I., O'Roak, B. J., Sanders, S. J., Ronemus, M., Krumm, N., Levy, D. et al. The contribution of de novo coding mutations to autism spectrum disorder. Nature 515 , 216-221 (2014)

2 De Rubeis, S., He, X., Goldberg, A. P., Poultney, C. S., Samocha, K., Ercument Cicek, A. et al. Synaptic, transcriptional and chromatin genes disrupted in autism. Nature $\mathbf{5 1 5}$, 209-215 (2014)

3 Iossifov, I., Ronemus, M., Levy, D., Wang, Z., Hakker, I., Rosenbaum, J. et al. De novo gene disruptions in children on the autistic spectrum. Neuron 74, 285-299 (2012).

4 Krumm, N., O'Roak, B. J., Shendure, J. \& Eichler, E. E. A de novo convergence of autism genetics and molecular neuroscience. Trends Neurosci. 37, 95-105 (2014)

5 Malhotra, D. \& Sebat, J. CNVs: harbingers of a rare variant revolution in psychiatric genetics. Cell 148, 1223-1241 (2012).

6 Neale, B. M., Kou, Y., Liu, L., Ma'ayan, A., Samocha, K. E., Sabo, A. et al. Patterns and rates of exonic de novo mutations in autism spectrum disorders. Nature 485 242-245 (2012)

7 O'Roak, B. J., Vives, L., Girirajan, S., Karakoc, E., Krumm, N., Coe, B. P. et al. Sporadic autism exomes reveal a highly interconnected protein network of de novo mutations. Nature 485, 246-250 (2012)

8 O'Roak, B. J., Deriziotis, P., Lee, C., Vives, L., Schwartz, J. J., Girirajan, S. et al. Exome sequencing in sporadic autism spectrum disorders identifies severe de novo mutations. Nat. Genet. 43, 585-589 (2011)

9 Sanders, S. J., Murtha, M. T., Gupta, A. R., Murdoch, J. D., Raubeson, M. J., Willsey, A. J. et al. De novo mutations revealed by whole-exome sequencing are strongly associated with autism. Nature 485, 237-241 (2012).

10 Ronemus, M., Iossifov, I., Levy, D. \& Wigler, M. The role of de novo mutations in the genetics of autism spectrum disorders. Nat. Rev. Genet. 15, 133-141 (2014).

11 Yasuda, Y., Hashimoto, R., Fukai, R., Okamoto, N., Hiraki, Y., Yamamori, H. et al. Duplication of the NPHP1 gene in patients with autism spectrum disorder and normal intellectual ability: a case series. Ann. Gen. Psychiatry 13, 22 (2014).

12 Yasuda, Y., Hashimoto, R., Ohi, K., Yamamori, H., Fujimoto, M., Umeda-Yano, S. et al. Cognitive inflexibility in Japanese adolescents and adults with autism spectrum disorders. World journal of psychiatry 4, 42-48 (2014).

13 Yasuda, Y., Hashimoto, R., Yamamori, H., Ohi, K., Fukumoto, M., Umeda-Yano, S. et al. Gene expression analysis in lymphoblasts derived from patients with autism spectrum disorder. Mol. Autism 2, 9 (2011).

14 Lord, C., Rutter, M. \& Le Couteur, A. Autism Diagnostic Interview-Revised: a revised version of a diagnostic interview for caregivers of individuals with possible pervasive developmental disorders. J. Autism Dev. Disord. 24, 659-685 (1994).

15 Yamada, A., Suzuki, M., Kato, M., Suzuki, M., Tanaka, S., Shindo, T. et al. Emotional distress and its correlates among parents of children with pervasive developmental disorders. Psychiatry Clin. Neurosci. 61, 651-657 (2007).

16 Wakabayashi, A., Tojo, Y., Baron-Cohen, S. \& Wheelwright, S. The Autism-spectrum quotient $(A Q)$ Japanese version: evidence from high-functioning clinical group and normal adults. Shinrigaku Kenkyu 75, 78-84 (2004).

17 Wechsler, D. Wechsler Adult Intelligence Scale-Third Edition Manual (The Psychological Corporation, San Antonio, TX, USA, 1997).
18 Wechsler, D. Wechsler Intelligence Scale for Children Third Edition Manual (The Psychological Corporation, New York, NY, USA, 1991).

19 Matsuoka, K., Uno, M., Kasai, K., Koyama, K. \& Kim, Y. Estimation of premorbid IQ in individuals with Alzheimer's disease using Japanese ideographic script (Kanji) compound words: Japanese version of National Adult Reading Test. Psychiatry Clin. Neurosci. 60, 332-339 (2006).

20 DePristo, M. A., Banks, E., Poplin, R., Garimella, K. V., Maguire, J. R., Hartl, C. et al. A framework for variation discovery and genotyping using next-generation DNA sequencing data. Nat. Genet. 43, 491-498 (2011).

21 Nakamura, K., Kodera, H., Akita, T., Shiina, M., Kato, M., Hoshino, H. et al. De Novo mutations in GNAO1, encoding a Galphao subunit of heterotrimeric G proteins, cause epileptic encephalopathy. Am. J. Hum. Genet. 93, 496-505 (2013).

22 Saitsu, H., Nishimura, T., Muramatsu, K., Kodera, H., Kumada, S., Sugai, K. et al. De novo mutations in the autophagy gene WDR45 cause static encephalopathy of childhood with neurodegeneration in adulthood. Nat. Genet. 45, 445-449 (2013).

23 Wang, K., Li, M. \& Hakonarson, H. ANNOVAR: functional annotation of genetic variants from high-throughput sequencing data. Nucleic Acids Res. 38, e164 (2010).

24 Narahara, M., Higasa, K., Nakamura, S., Tabara, Y., Kawaguchi, T., Ishii, M. et al. Large-scale East-Asian eQTL mapping reveals novel candidate genes for LD mapping and the genomic landscape of transcriptional effects of sequence variants. PLOS ONE 9, e100924 (2014).

25 Liu, H., Nakazawa, T., Tezuka, T. \& Yamamoto, T. Physical and functional interaction of Fyn tyrosine kinase with a brain-enriched Rho GTPase-activating protein TCGAP. J. Biol. Chem. 281, 23611-23619 (2006).

26 Kawa, S., Ito, C., Toyama, Y., Maekawa, M., Tezuka, T., Nakamura, T. et al. Azoospermia in mice with targeted disruption of the Brek/Lmtk2 (brain-enriched kinase/lemur tyrosine kinase 2) gene. Proc. Natl Acad. Sci. USA 103, 19344-19349 (2006).

27 Hewinson, J., Paton, J. F. \& Kasparov, S. Viral gene delivery: optimized protocol for production of high titer lentiviral vectors. Methods Mol. Biol. 998, 65-75 (2013).

28 Delawary, M., Tezuka, T., Kiyama, Y., Yokoyama, K., Inoue, T., Hattori, S. et al. NMDAR2B tyrosine phosphorylation regulates anxiety-like behavior and CRF expression in the amygdala. Mol. Brain 3, 37 (2010).

29 Clagett-Dame, M., McNeill, E. M. \& Muley, P. D. Role of all-trans retinoic acid in neurite outgrowth and axonal elongation. J. Neurobiol. 66, 739-756 (2006).

30 Mashimo, T., Hadjebi, O., Amair-Pinedo, F., Tsurumi, T., Langa, F., Serikawa, T. et al. Progressive Purkinje cell degeneration in tambaleante mutant mice is a consequence of a missense mutation in HERC1 E3 ubiquitin ligase. PLoS Genet. 5, e1000784 (2009).

31 Dong, X., Shen, K. \& Bulow, H. E. Intrinsic and Extrinsic Mechanisms of Dendritic Morphogenesis. Annu. Rev. Physiol. 77, 271-300 (2014).

32 Zikopoulos, B. \& Barbas, H. Changes in prefrontal axons may disrupt the network in autism. J. Neurosci. 30, 14595-14609 (2010)

33 Mukaetova-Ladinska, E. B., Arnold, H., Jaros, E., Perry, R. \& Perry, E. Depletion of MAP2 expression and laminar cytoarchitectonic changes in dorsolateral prefrontal cortex in adult autistic individuals. Neuropathol. Appl. Neurobiol. 30, 615-623 (2004).

34 Penzes, P., Cahill, M. E., Jones, K. A., VanLeeuwen, J. E. \& Woolfrey, K. M. Dendritic spine pathology in neuropsychiatric disorders. Nat. Neurosci. 14, 285-293 (2011).

35 Srivastava, D. P. Woolfrey, K. M. Jones, K. A Anderson, C. T., Smith, K. R. Russell, T. A. et al. An autism-associated variant of Epac2 reveals a role for Ras/Epac2 signaling in controlling basal dendrite maintenance in mice. PLoS Biol. 10, e1001350 (2012).

36 de Anda, F. C., Rosario, A. L., Durak, O., Tran, T., Graff, J., Meletis, K. et al. Autism spectrum disorder susceptibility gene TAOK2 affects basal dendrite formation in the neocortex. Nat. Neurosci. 15, 1022-1031 (2012).

37 Kwon, C. H., Luikart, B. W., Powell, C. M., Zhou, J., Matheny, S. A., Zhang, W. et al. Pten regulates neuronal arborization and social interaction in mice. Neuron 50 , 377-388 (2006).

38 Murcia, C. L., Gulden, F. \& Herrup, K. A question of balance: a proposal for new mouse models of autism. Int. J. Dev. Neurosci. 23, 265-275 (2005).

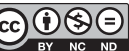

This work is licensed under a Creative Commons Attribution-NonCommercial-NoDerivs $\quad 4.0$ International License. The images or other third party material in this article are included in the article's Creative Commons license, unless indicated otherwise in the credit line; if the material is not included under the Creative Commons license, users will need to obtain permission from the license holder to reproduce the material. To view a copy of this license, visit http://creativecommons.org/licenses/bync-nd/4.0/

Supplementary Information accompanies the paper on Journal of Human Genetics website (http://www.nature.com/jhg) 